\title{
Anterolateral Papillary Muscle Rupture Caused by Brucella Endocarditis
}

\author{
Feridoun Sabzi ${ }^{1}$; Nasrin Javid ${ }^{1}$; Reza Faraji ${ }^{1{ }^{*}}$ \\ ${ }^{1}$ Department of Cardiovascular Surgery, Imam Ali Heart Center, Kermanshah University of Medical Sciences, Kermanshah, IR Iran \\ ${ }^{*}$ Corresponding author: Reza Faraji, Department of Cardiovascular Surgery, Imam Ali Heart Center, Kermanshah University of Medical Sciences, Kermanshah, IR Iran. \\ E-mail: r.faraji61@gmail.com
}

Received: April 16, 2014; Revised: June 10, 2014; Accepted: August 20, 2014

\begin{abstract}
Introduction: Brucellosis is a common zoonosis in Iran, transmitted to humans by direct contact with infected animal materials like fleece, animal excrement, leather, butchery or by the consumption of contaminated animal products (e.g. fresh cheese, unpasteurized milk or dairy products, and raw meat and raw liver that is consumed semi-roasted by kebab sellers in Iran.

Case Presentation: A 21-year-old woman presented with respiratory failure due to pulmonary edema from severe acute mitral valve regurgitation in the setting of acute Brucella endocarditis. She was found to have a ruptured anterolateral papillary muscle from necrosis caused by corroding large vegetations on the anterior mitral valve leaflet.

Conclusions: This occurrence is an exceptionally rare phenomenon, in Brucella mitral endocarditis. Urgent transesophageal echocardiography (TEE) revealed large mobile vegetation on the anterior papillary muscle with severe mitral valve regurgitation. The patient underwent mitral valve replacement. During valve replacement surgery, anterior mitral valve vegetation and rupture of the AL-PM were confirmed. We also considered that prolonged post-operative antibiotic treatment was indicated in our patient to protect the newly implanted prosthetic valves.
\end{abstract}

Keywords: Endocarditis; Brucellosis; Papillary Muscle Rupture

\section{Introduction}

Brucellosis is a common zoonosis in Iran, transmitted to humans by direct contact with infected animal materials like fleece, animal excrement, leather, butchery or by the consumption of contaminated animal products (e.g. fresh cheese, unpasteurized milk or dairy products, and raw meat and raw liver that is consumed semi-roasted by kebab sellers in Iran [1]. A case is described of Brucella endocarditis on the mitral valve causing papillary muscle rupture. It was treated by a combined medical and surgical approach. Because papillary muscle rupture by Brucella endocarditis is an exceedingly rare complication, general treatment recommendations do not exist for this potentially fatal complication. Therapeutic strategies in Brucella endocarditis are discussed.

\section{Case Presentation}

A 21-year-old woman presented with a two weeks history of fever, chills, night sweating, and arthralgia. Her medical history was important as she was living in a rural village, consuming fresh cheese and unpasteurized milk. There was no history of illicit drug use, autoimmune disease, HIV or other immune-compromising disease. The patient was febrile, and physical examination revealed an ejection systolic murmur over the mi- tral area caused by use mitral valve regurgitation (MR), which had been confirmed by trans-thoracic echocardiography (TTE). Blood cultures and Brucella test were obtained and she was admitted to the cardiac care unit (CCU). The patient's general condition deteriorated within 24 hours because of respiratory failure followed by hypotension and acute renal failure which required intubation, mechanical ventilation, and initiation of inotropic drugs. Electrocardiography showed ischemic change with inferior ST-segment elevation. Urgent transesophageal echocardiography (TEE) revealed large mobile vegetation on the anterior papillary muscle (Figure 1) with severe mitral valve regurgitation. More importantly, TEE also detected large vegetation on the anterior mitral valve leaflet (Figure 2) along with a hypokinetic lateral wall of the left ventricle and severe mitral valve regurgitation (Figure 3 ).

Cardiac catheterization revealed a normal coronary artery rule out occlusion of any branch of the dominant right coronary artery due to embolic mitral vegetation. The patient underwent mitral valve replacement. During valve replacement surgery, anterior mitral valve vegetation and rupture of the AL-PM (Figure 4) were confirmed. Large vegetations were seen on the mitral valve.

Copyright (c) 2015, Zahedan University of Medical Sciences. This is an open-access article distributed under the terms of the Creative Commons Attribution-NonCommercial 4.0 International License (http://creativecommons.org/licenses/by-nc/4.0/) which permits copy and redistribute the material just in noncommercial usages, provided the original work is properly cited. 


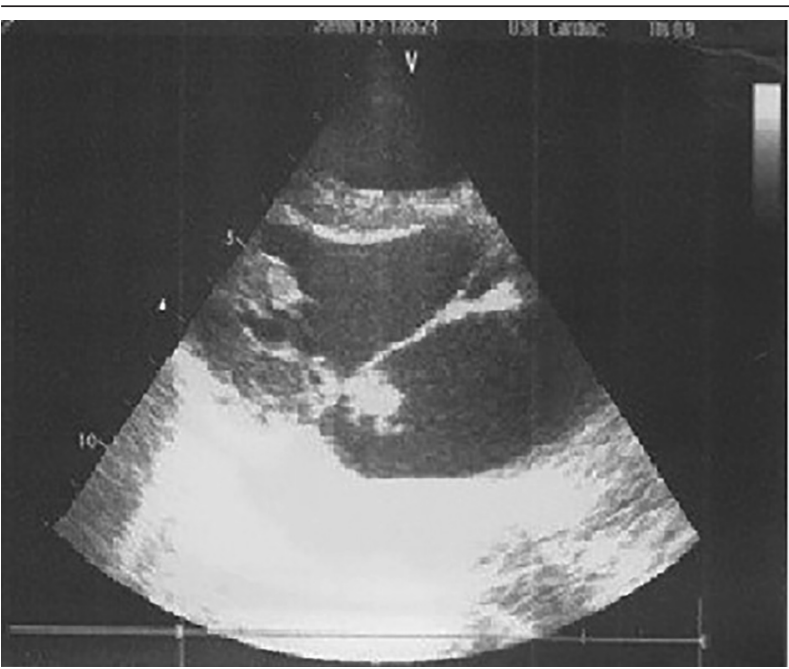

Figure 1. Parasternal Long Axis View Shows Vegetation on Papillary Muscle Extended to Left Atrial Side

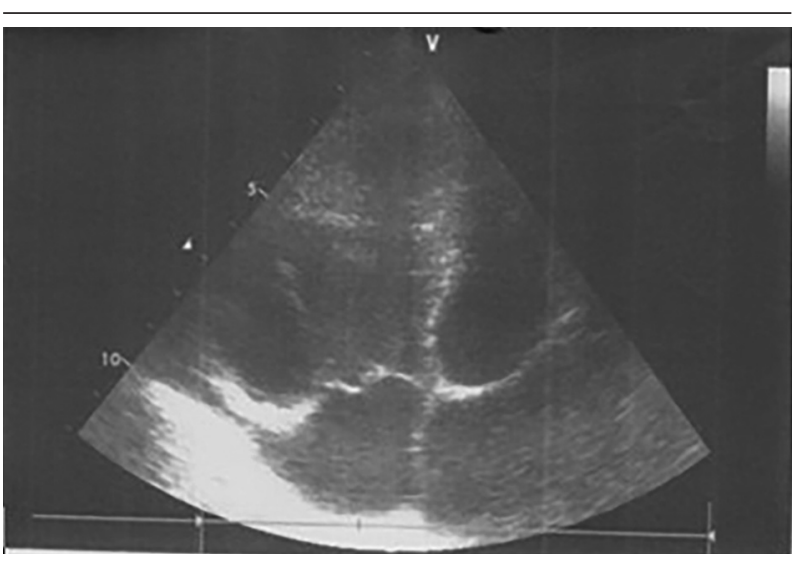

Figure 2. Four Chamber View Showing Large, Irregular-Shaped Vegetation Attached to the Anterior Mitral Valve Leaflet

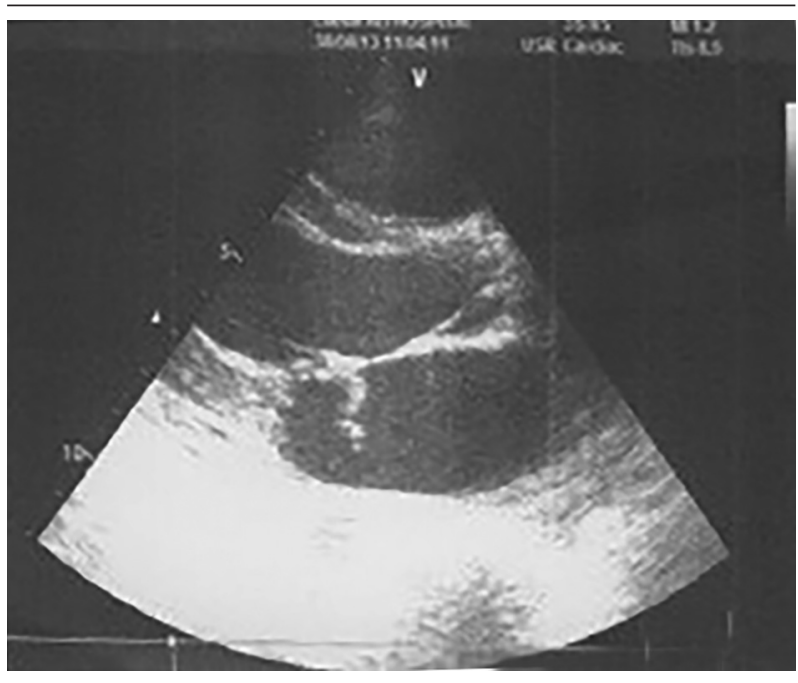

Figure 3. Long Axis Parasternal Views Showing Ruptured Head of the Anterolateral Papillary Muscle in the Left Atrium With Mitral Valve Regurgitation (LA)

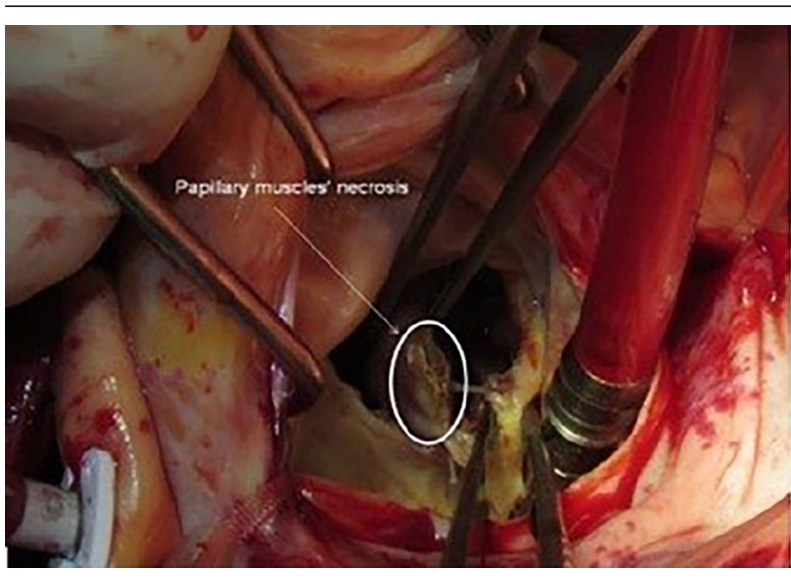

Figure 4. Intra-Operative Photograph Showing Ruptured Anterolateral Papillary Muscle (Arrow) With Ruptured Chords Resting Inside the Left Atrium

\section{Discussion}

In the study by Colmenero et al. cardiac involvement such as, pericarditis, myocarditis and endocarditis occurred in fewer than $2 \%$ of all case and demonstrated that endocarditis has a low frequency, but the majority of deaths due to Brucella endocarditis are related to cardiac failure as a consequence of valve regurgitation or myocardial infarction or myocarditis [2]. In the study by Jacobs et al. [3], more than half of the patients with Brucella endocarditis had underlying valvular damage like perforation of leaflet but no case of papillary muscle rupture was reported in their series. However, a comprehensive Medline search revealed 43 cases of Brucella mitral valve endocarditis up to 2013. Mitral regurgitation as a result of papillary muscle necrosis has been described only in one case of embolic vegetation to left circumflex artery (LCX) branches but direct necrosis of papillary muscle caused by vegetation was not reported [3]. In endemic areas, however Brucella has established a low incidence among the causative agents of native valve endocarditis. However, to the best of our knowledge, no case of papillary muscle necrosis as a result of vegetation of Brucella endocarditis on the mitral valve has been reported. Quiroga et al. for the first time described a case of mitral leaflet perforation following Brucella endocarditis [4].

Jeroudi et al. reported an interesting case of ruptured chordae tendineae and flail leaflets of mitral apparatus caused by large vegetation on the mitral valve [5]. Farid and Trabolsi reported one case of Brucella native valve endocarditis that has been cured by medical treatment alone [6]. In Chan and Hardiman's study, Brucella was still found in cultures of infected valves after 8 weeks of adequate antibiotic therapy [7].

In the study by Cisneros et al. relapses occurred two years after a supposed cure, and they offered that an attempt at medical treatment without surgical intervention absolutely required careful monitoring under 
therapy, as well as strict and prolonged clinical and echocardiographic surveillance [8]. Echocardiography follow-up of Brucellosis patients with a history of valvular heart disease is mandatory [8]. Brucella endocarditis in adults, in most cases either ends fatally or requires emergency valve replacement, despite medical treatment using a combination of oral tetracycline for three weeks, together with intramuscular streptomycin for 2 weeks. There is no general recommendation by WHO for a postoperative course of disease or how long antimicrobial agents should be administered after valve replacement, but WHO, recommended clinical, serological and microbiological findings to help to decide whether antibiotic treatment should be continued or not. We used the normalization of anti-Brucella titers as recommended by Jeroudi et al. to be the end point of an effective antibiotic therapy in the post-operative period [5]. We also considered that prolonged post-operative antibiotic treatment was indicated in our patient to protect the newly implanted prosthetic valves.

Papillary muscle rupture is exceedingly rare complication of mitral valve Brucella endocarditis pre-operative long-term antibiotic treatment of Brucella endocarditis before surgery was insufficient to eradicate the infection in our patient with large mobile vegetation on mitral valve. Mitral valve replacement seems to be mandatory in the management of Brucella mitral valve endocarditis complicated by severe MR and clinical sign and symptom of pulmonary edema. However, the timing of surgery remains controversial and should be based on the patient's clinical condition.

\section{Acknowledgements}

Efforts of Dr. Nikokar and Dr. Qasemzadeh are highly appreciated.

\section{References}

1. Arslan H, Korkmaz ME, Kart H, Gul C. Management of brucella endocarditis of a prosthetic valve. J Infect. 1998;37(1):70-1.

2. Colmenero JD, Reguera JM, Martos F, Sanchez-De-Mora D, Delgado M, Causse M, et al. Complications associated with Brucella melitensis infection: a study of 530 cases. Medicine (Baltimore). 1996;75(4):195-211.

3. Jacobs F, Abramowicz D, Vereerstraeten P, Le Clerc JL, Zech F, Thys JP. Brucella endocarditis: the role of combined medical and surgical treatment. Rev Infect Dis. 1990;12(5):740-4.

4. Quiroga J, Miralles A, Farinola T, Obi C, Granados J, Fontanillas C, et al. Surgical treatment of Brucella endocarditis. Cardiovasc Surg. 1996;4(2):227-30.

5. Jeroudi MO, Halim MA, Harder EJ, Al-Siba'i MB, Ziady G, Mercer EN. Brucella endocarditis. Br Heart J. 1987;58(3):279-83.

6. Farid Z, Trabolsi B. Successful treatment of two cases of Brucella endocarditis with rifampicin. Br Med J. 1985;291:110.

7. Chan R, Hardiman RP. Endocarditis caused by Brucella melitensis. Med J Aust. 1993;158(9):631-2.

8. Cisneros JM, Pachon J, Cuello JA, Martinez A. Brucella endocarditis cured by medical treatment. J Infect Dis. 1989;160(5):907. 